\title{
Potenciais Interações Medicamento-Alimento e Medicamento-Planta Medicinal em Farmácias Distritais de um Município do Seridó Potiguar
}

\author{
Potential Drug-Food and Drug-Medicinal Plant interactions in District \\ Pharmacies at a Seridó Potiguar county
}

Recebido em: 18/12/2020 Aceito em: 12/07/2021
Lucas Nóbrega de OLIVEIRA; Almária Mariz BATISTA Escola Multicampi de Ciências Médicas do Rio Grande do Norte. Universidade Federal do Rio Grande do Norte (EMCM/UFRN). Av. Dr. Carlindo Dantas, 540, $2^{\circ}$ andar, CEP 59300-000. Caicó, RN, Brasil.

E-mail:almariamariz@yahoo.com.br

\section{ABSTRACT}

This study evaluated possible drug-food and drug-medicinal plant interactions involving antidiabetics and antihypertensive medication on primary health care in the city of Caicó, RN, Brazil, through a descriptiveexploratory cross-sectional study, with a quantitative approach involving two district pharmacies in Caicó, RN. The object of study consisted of duplicated drug prescriptions of medication dispensed during the period of August to December 2019. Micromedex, Drugs.com and Up to Date, were used to assess drugfood interactions in addition to the medicinal informational leaflets provided by the National Health Surveillance Agency (Anvisa). Due to the lack of databases referring to medicinal plants, the evaluation of drug-medicinal plant interactions was based on articles indexed via CAPES and Google Scholar journals. Among 569 analyzed prescriptions, 11 contained antihypertensive and 3 contained anti-diabetic drugs. Among these prescriptions, 10 contained important interactions; 6 were considered negative (1 mild, 4 moderate and 1 moderate to severe) and 4 positives. Furthermore, we observed that 7 drugs were involved in drug-medicinal plant interactions, which were considered positive or negative depending on the dosage of the medication and medicinal plant. Therefore, it is of utmost importance to develop more studies on this topic as well as to raise awareness and instrumentalize health professionals, and users, about the proper management of pharmacological therapy in order to enjoy the beneficial interactions and avoid the negative impacts on therapeutic effectiveness and patient safety.

Keywords: antihypertensive; antidiabetics; drug interactions; primary care.

\section{RESUMO}

Esse estudo teve como objetivo avaliar possíveis interações do tipo medicamento-alimento e medicamentoplanta medicinal envolvendo antidiabéticos e anti-hipertensivos na atenção primária à saúde de Caicó, RN, Brasil. O estudo foi do tipo transversal, de caráter descritivo-exploratório, com abordagem quantitativa em duas farmácias distritais de Caicó, RN. O objeto de estudo constituiu-se de todas as segundas vias das 
prescrições de medicamentos elaboradas durante o período de agosto a dezembro de 2019. Para avaliação das interações medicamento-alimento foram utilizadas as bases de dados Micromedex, Drugs.com e Up to Date, além do bulário disponibilizado pela Agência Nacional de Vigilância Sanitária (Anvisa). Pela carência de bases de dados referentes a plantas medicinais, a avaliação das interações medicamento-planta medicinal baseou-se em artigos indexados via Periódicos Capes e Google Scholar. Foram analisadas 569 prescrições, nas quais constavam 11 anti-hipertensivos e 3 antidiabéticos. Foi constatado que 10 medicamentos apresentavam interações significativamente importantes, entre elas, 6 consideradas negativas (1 leve, 4 moderadas e 1 moderada a grave) e 4 positivas. Ademais, foi possível 7 medicamentos estavam envolvidos em interações do tipo medicamento-planta medicinal e todas foram consideradas positivas/negativas a depender da dose e frequência de administração do medicamento e da planta medicinal. Portanto, é de suma importância o desenvolvimento de mais estudos sobre este tema bem como sensibilização e instrumentalização de profissionais de saúde e usuários acerca do manejo adequado da terapia farmacológica, a fim de usufruir das interações benéficas bem como evitar impactos negativos sobre a efetividade terapêutica e a segurança do paciente que algumas interações podem causar.

Palavras-chaves: anti-hipertensivos; antidiabéticos; interações medicamentosas; atenção básica.

\section{INTRODUÇÃO}

A Organização Mundial de Saúde (OMS) estima que cerca de $70 \%$ de todas as mortes do mundo são causadas por doenças crônicas não transmissíveis (DCNT), como diabetes e hipertensão (1). No Brasil, este dado apresenta magnitude maior, sendo $75 \%$ de mortes causadas por DCNT (2). Diabetes mellitus é um importante e crescente problema de saúde para todos os países, independentemente do seu grau de desenvolvimento. Esta doença pode causar inúmeras complicações como retinopatia, nefropatia, neuropatia e doenças coronariana, cerebrovascular e arterial periférica. O tratamento medicamentoso inclui hipoglicemiantes orais e insulina (3). Hipertensão arterial tem alta prevalência e é um dos principais fatores de risco para doenças cardiovasculares e renais, tendo como fatores de risco idade, sexo, etnia, ingesta de álcool, sedentarismo e fatores genéticos. $\mathrm{O}$ tratamento medicamentoso inclui bloqueadores de canais de cálcio, betabloqueadores, diuréticos, vasodilatadores diretos, inibidores da enzima conversora de angiotensina, bloqueadores de receptores AT1 da angiotensina II, inibidores diretos da renina. Seu controle em todo mundo é difícil, devido ser frequentemente assintomática, o que dificulta a adesão aos cuidados (4).

Diante desses agravos, o tratamento medicamentoso destas DCNT deve ser associado a trata- mento não-medicamentoso, sendo este voltado à mudança no estilo de vida, ou seja, prática regular de exercício físico, alimentação saudável, gerenciamento de estresse, redução do consumo de bebidas alcoólicas e eliminação do tabagismo (5).

A maioria dos medicamentos é administrada via oral, pois é uma fácil via de administração e a mucosa digestiva oferece boa absorção para vários medicamentos. Entretanto, isto aumenta o risco de interações medicamentosas, como interação medicamento-alimento (6), a qual ocorre quando um fármaco/nutriente altera a eficácia de determinado medicamento ou interfere no estado nutricional do indivíduo (7). Estas interações podem causar diminuição de efeito terapêutico ou toxicidade. Por outro lado, podem trazer benefícios como acréscimo ou maior duração de efeito terapêutico ou até diminuição de efeitos indesejáveis (8).

A população utiliza terapias complementares, como plantas medicinais, para tratar de algumas enfermidades (9). Entretanto, muitas plantas medicinais podem interagir com medicamentos e causar efeitos potencialmente perigosos, a quem faz este uso, pois apresentam efeitos farmacológicos, dependendo do tipo de preparação/finalidade (10).

Grande parte das interações medicamento-alimento é de difícil reconhecimento, podendo causar inúmeros prejuízos na ação tanto do medicamento quanto do alimento ou em ambas, como é o caso de 
alprazolam ou clonazepam, que, quando administrados com cafeína pode diminuir efeitos ansiolítico e sedativo do fármaco. Para que situações como esta não aconteçam é necessário que se observe, primeiramente, dose e tempo de administração dos medicamentos em relação à refeição, pois são os principais fatores determinantes para que aconteçam estes tipos de interação (11).

Nessa perspectiva, alguns alimentos/nutrientes apresentam maior tendência a interações, como os ricos em cálcio, que interagem com alguns medicamentos causando quelação de fármacos ou depleção da absorção deste íon. Isto suscita a necessidade de entender os principais alimentos/nutrientes que podem causar efeitos negativos ou positivos em relação aos fármacos (12).

A maioria das interações não são evidenciadas ou notificadas, devido, principalmente, à escassez de estudos nesta área, somando-se a falta de conhecimento dos profissionais de saúde e carência de farmacêutico nas equipes de saúde, contribuindo para que, muitas vezes, estas interações não sejam constatadas nem sanadas da melhor forma possível, podendo comprometer a segurança do paciente (13).

Outrossim, deve-se levar em consideração a prevalência de DCNT, principalmente, diabetes e hipertensão, doenças relacionadas a morbimortalidade e polifarmácia, sendo assim, de suma importância o desenvolvimento de estudos que tratem desse tema, a fim de sensibilizar gestores, profissionais de saúde e usuários. Nessa perspectiva, este estudo se propõe a avaliar possíveis interações do tipo medicamento-alimento e medicamento-planta medicinal envolvendo antidiabéticos e anti-hipertensivos na atenção primária à saúde de Caicó, RN.

\section{MÉTODO}

O estudo foi do tipo transversal, de caráter descritivo-exploratório, com abordagem quantitativa, em duas farmácias distritais, uma localizada na zona central e outra na zona oeste do município de Caicó, RN, considerado o $5^{\circ}$ maior do Rio Grande do Norte em área territorial, com $1228,584 \mathrm{~km}^{2}$. A população estimada é de 68.343 habitantes (14).
Além disso, o município apresenta 24 Equipes de Estratégias de Saúde da Família (ESF), sendo 21 destas na zona urbana e 3 na zona rural, além de 5 farmácias distritais e 1 Central de Abastecimento Farmacêutico (CAF).

O objeto de estudo constituiu-se de todas as segundas vias das prescrições de medicamentos elaboradas de agosto a dezembro de 2019, arquivadas nestas 2 farmácias distritais, independente do serviço de saúde de onde eram provenientes, as quais apresentavam antidiabéticos e anti-hipertensivos prescritos por médicos. Além disso, tendo em vista que diabetes e hipertensão são doenças crônicas e, por este motivo, existe o processo de renovação de prescrições, considerou-se 1 prescrição de cada paciente, ou seja, as repetidas foram excluídas do estudo.

Para avaliação das interações medicamento-alimento e foram utilizadas as bases de dados $\mathrm{Mi}$ cromedex (15), Drugs.com (16) e Up to Date (17), além do bulário disponibilizado pela Agência Nacional de Vigilância Sanitária (ANVISA) (18). Pela carência de bases de dados referentes a plantas medicinais, a avaliação das interações medicamento-planta medicinal baseou-se em artigos indexados via Periódicos Capes e Google Scholar.

Os dados foram tabulados e analisadas via Microsoft Excel, por meeio de estatística descritiva, via gráficos e frequências absoluta e relativa.

O estudo foi aprovado pelo Comitê de Ética do Hospital Universitário Onofre Lopes (HUOL), sob $\mathrm{n}^{\mathrm{o}}$ de protocolo 64367517.3.0000.5292.

\section{RESULTADOS E DISCUSSÃO}

Foram analisadas 569 prescrições, em que $100 \%$ foram provenientes de unidades básicas de saúde, nas quais constavam, ao todo, 11 tipos de anti-hipertensivos e 3 tipos de antidiabéticos. Dentre estas, 343 apresentavam apenas anti-hipertensivos $(60,3 \%), 133$ continham apenas antidiabéticos $(23,4 \%)$ e 93 apresentavam estes 2 tipos de medicamentos (16,3\%) (Figura 1). Hipertensão e diabetes são consideradas DCNT que mais acometem a população brasileira, podendo, muitas vezes, incidir no mesmo indivíduo (19). 
Dentre os anti-hipertensivos prescritos, hidroclorotiazida foi o mais prevalente, encontrado em 217 prescrições $(63,2 \%)$, seguido de losartana em 158 prescrições (46\%) e enalapril em 129 prescrições $(37,6 \%)$ (Figura 2). Estes dados corroboram estudo realizado em Maringá, PR, o qual constatou hidroclorotiazida como anti-hipertensivo mais utilizado pelos usuários da atenção básica deste município, seguido de losartana, atenolol, anlodipino, enalapril, captopril, propranolol e nifedipino (20).

Figura 1. Distribuição das classes farmacológicas presentes nas 569 prescrições avaliadas em Caicó, RN, 2019.

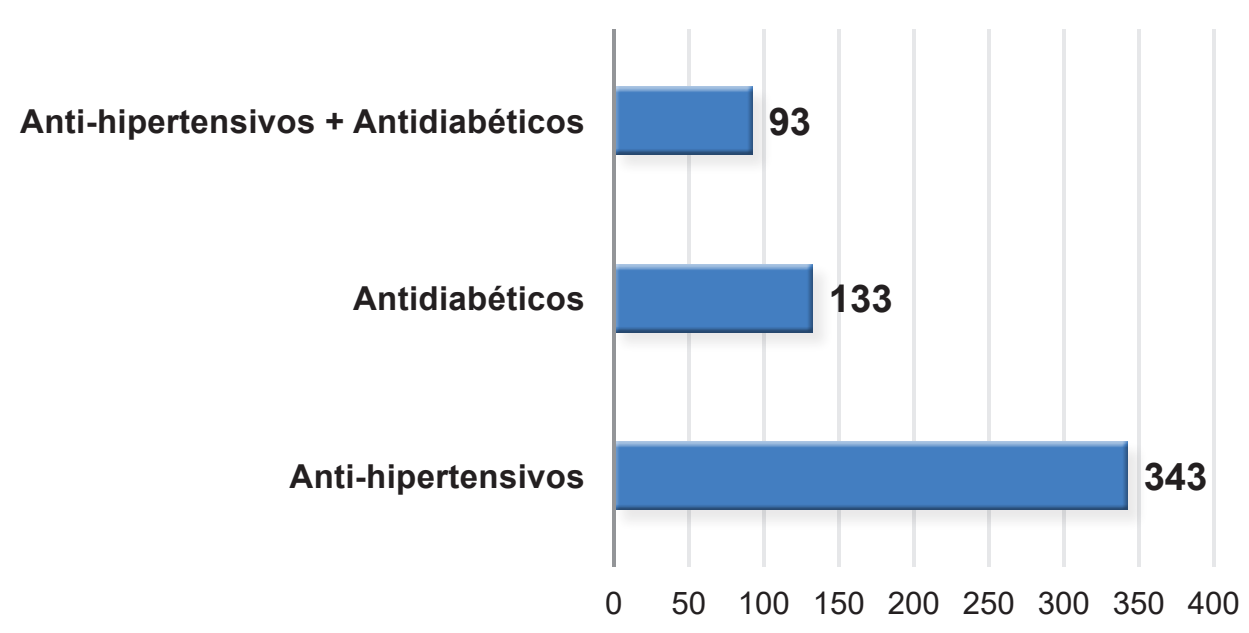

Figura 2. Distribuição dos anti-hipertensivos presentes nas 569 prescrições avaliadas em Caicó, RN, 2019.

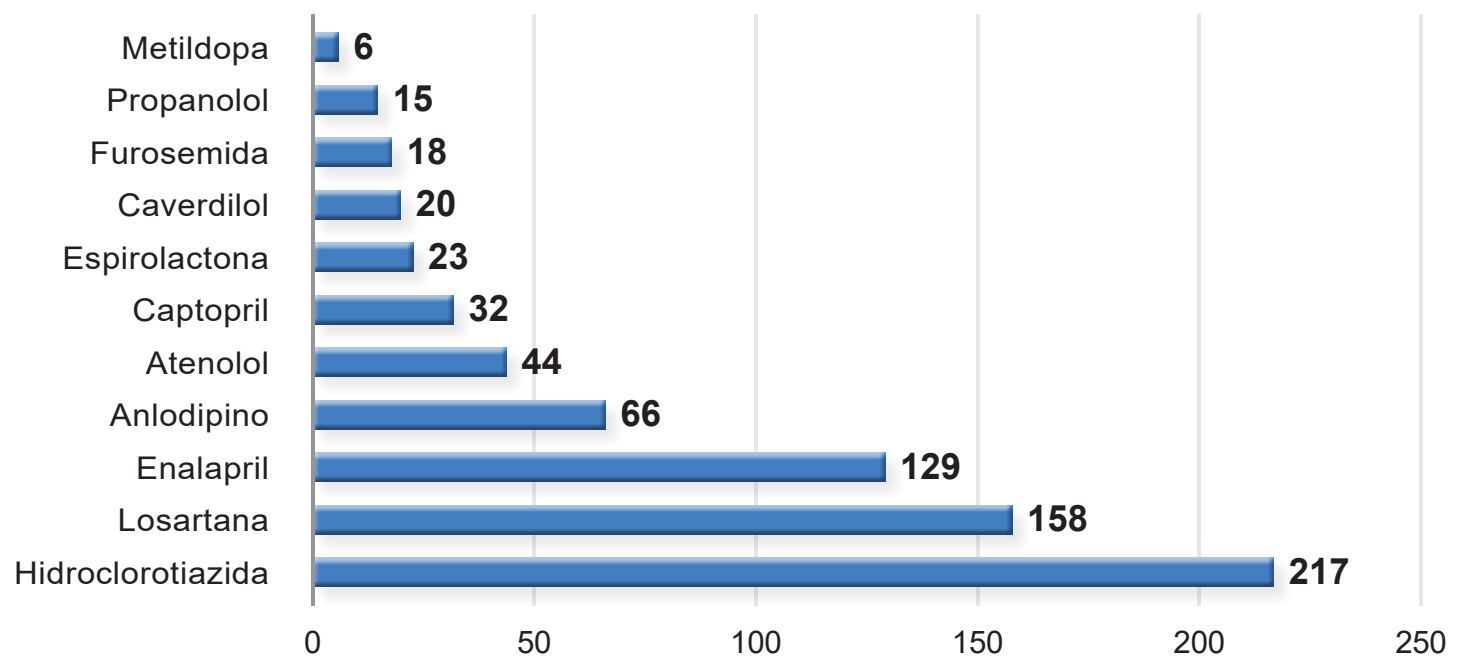

Quanto aos antidiabéticos prescritos, predominou a metformina, encontrada em 117 prescrições $(87,9 \%)$ (Figura 3). A avaliação de prescrições em UBS de Barbosa Ferraz, PR constatou prevalência da associação metformina + glibenclamida $(33,3 \%)$, além da monoterapia com metformina $(16,67 \%)$ ou glibenclamida $(13,33 \%)(21)$.
Nas prescrições analisadas foi constatada a presença de 15 especialidades médicas, com predomínio de Clínico Geral como prescritor, no caso, 441 prescrições $(77,5 \%)$. Além disso, penas 8 prescrições $(1,4 \%)$ foram elaboradas por cardiologistas e 7 por endocrinologistas (1,2\%) (Figura 4). Outrossim, apenas 7 prescrições $(1,2 \%)$ foram elabo- 
radas por médicos de família e comunidade (MFC), o que pode apontar carência desta especialidade na atenção primária de Caicó. Apenas $2 \%$ dos médicos do Brasil são especialistas nesta área e, apesar da atenção primária não ser campo exclusivo desta especialidade, é de suma importância sua colaboração para qualificação deste nível de atenção, pois sua formação é voltada a abordagem do pa- ciente, comunicação e conteúdo de humanidades relacionadas à saúde, a fim de proporcionar planos terapêuticos mais cuidadosos e singulares que aliviem seu sofrimento $(22,23)$. Um estudo realizado em Curitiba, PR, mostrou que usuários atendidos por MFC apresentaram expressiva redução do número de internamentos por insuficiência cardíaca da região (24).

Figura 3. Distribuição dos antidiabéticos presentes nas 569 prescrições avaliadas em Caicó, RN, 2019.

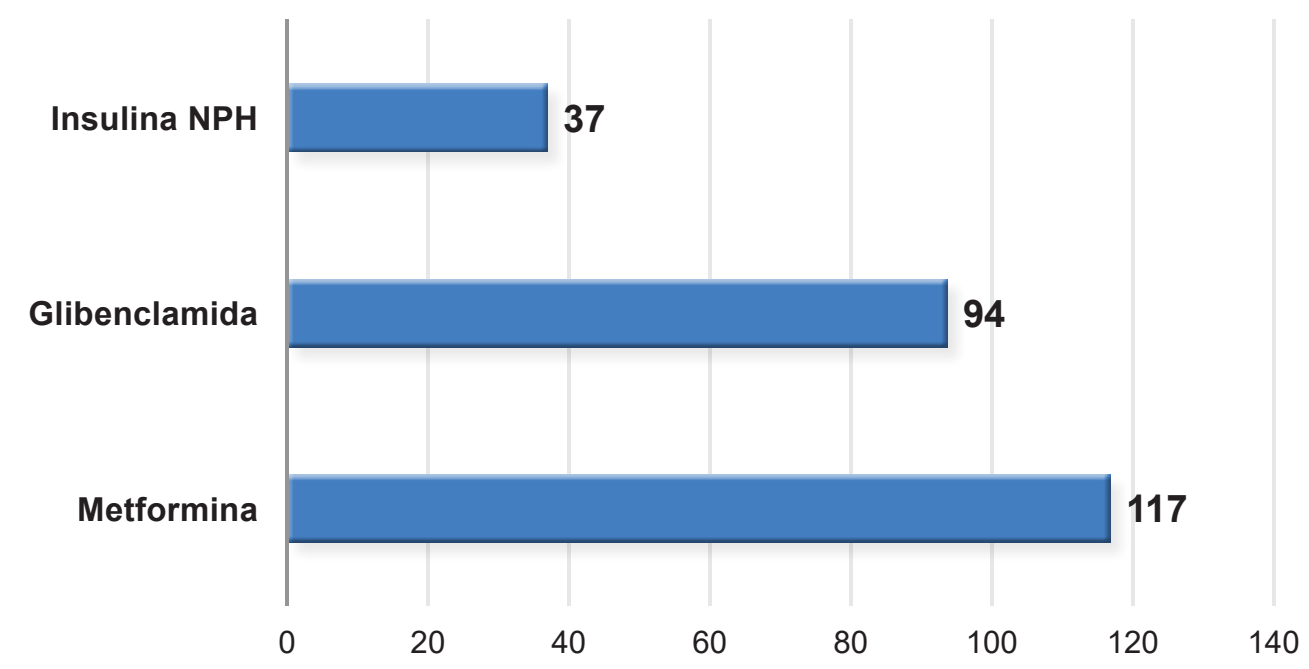

Figura 4. Distribuição das especialidades médicas nas 569 prescrições analisadas em Caicó, RN, 2019.

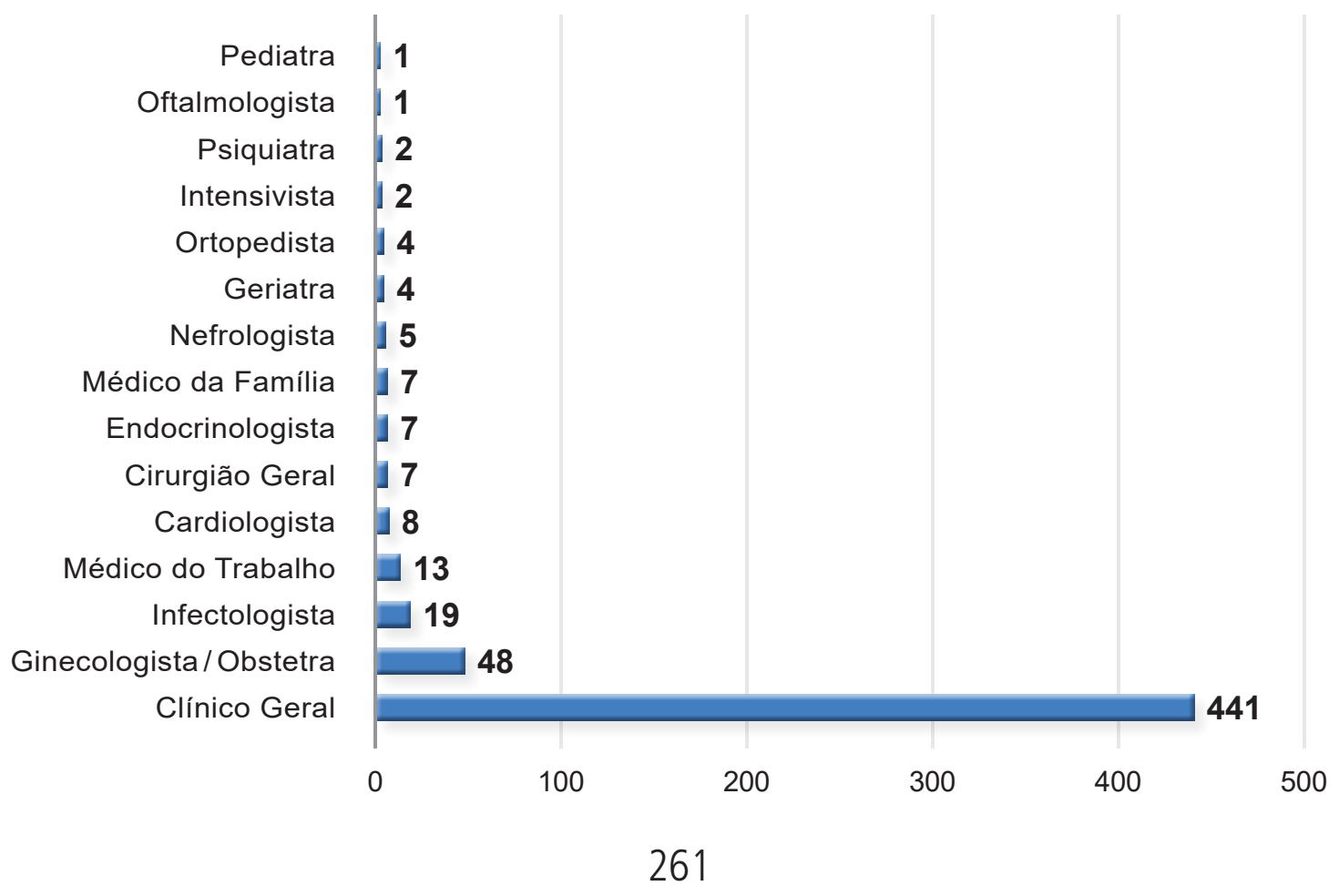


O MFC tem sua abordagem centrada na pessoa e na família, no contexto social, na prevenção e nos riscos populacionais, diferentemente do generalista, que, apesar de ter que conhecer todo campo do conhecimento médico, em qualquer campo da Medicina o especialista conhece mais. Além disso, o MFC necessita de inúmeras problematizações de sua prática clínica, uma vez que esta não deve se restringir a aspectos biológicos do ser humano (25).

Ademais, apenas 15 prescrições (2,64\%) foram elaboradas por cardiologistas e endocrinologistas, o que pode remeter a carência de avaliação e acompanhamento mais específico dos usuários do Sistema Único de Saúde (SUS) por estas especialidades, ponto que requer investigação mais específica, uma vez que todas as prescrições analisadas neste estudo foram provenientes de serviços de atenção primária (UBS), nenhuma da atenção secundária.

Quanto às interações medicamento-alimento, dos 14 fármacos analisados, 10 (71,4\%) estavam envolvidos em interações significativamente importantes (Quadro 1), sendo 9 anti-hipertensivos e 1 antidiabético. Todos estão disponíveis na versão atual da Relação Municipal de Medicamentos Essenciais (REMUME 2014) (26).

As interações medicamentosas podem ter características positivas, ou seja, proporcionar alguma vantagem terapêutica, ou negativas, podendo causar riscos à segurança do usuário ou à efetividade terapêutica. Esta segunda característica pode ser classificada de acordo com a gravidade, sendo considerada de risco alto, quando o risco supera o benefício; risco moderado, quando só é indicado o uso em condições especiais e risco leve, quando é necessário avaliar o risco e considerar um medicamento alternativo (16). Neste estudo, dentre as 6 interações de caráter negativo, apenas 1 foi considerada leve, 4 moderadas e 1 moderada à grave.

Alguns anti-hipertensivos como propranolol, carvedilol e nifedipino, quando administrados na presença de alimentos, apresentam nível de segurança melhor, pois o alimento age como protetor da mucosa estomacal, além de contribuir para dimi- nuição de náuseas e vômitos (27). Por outro lado, alimentos hiperproteicos como carne, ovo e laticínios podem ocasionar aumento da biodisponibilidade do propranolol $(15,16,18)$.

No que concerne ao captopril, quando administrado na presença de alimentos ricos em ferro, pode haver produção de complexos insolúveis que impedem significativamente a absorção do fármaco, afetando diretamente a efetividade do tratamento $(13,28)$. Assim, o intervalo de tempo entre a administração do medicamento e a ingestão de alimentos é imprescindível para o sucesso do tratamento (29).

Durante o período gestacional ocorre a suplementação de várias substâncias, entre elas, o ácido fólico, o qual tem papel fundamental na formação do tubo neural no feto (30). Metildopa é considerado fármaco de primeira escolha para tratamento da hipertensão gestacional (15). Porém, foi possível constatar neste estudo que, na presença de folato, um metabólito secundário do ácido fólico, a metildopa pode ter sua absorção prejudicada, afetando diretamente a efetividade do tratamento.

Enalapril, na presença de alimentos ricos em potássio, pode causar hiperpotassemia decorrente da diminuição da excreção de potássio, proporcionada pelo efeito inibitório da aldosterona, o que pode resultar em fraqueza muscular, toxicidade cardíaca e arritmias, sendo uma interação fármaco-nutriente de caráter negativo (31).

Sobre hidroclorotiazida e espironolactona, quando administrados na presença de alimentos em geral, têm sua biodisponibilidade aumentada; assim, estes devem ser ingeridos, preferencialmente, durante as refeições (28). Quanto à furosemida, isto se inverte; na presença de alimentos pode haver diminuição significativa de até $45 \%$ de fármaco absorvido, resultando em diminuição da resposta diurética $(8,32)$.

Entre os antidiabéticos analisados, apenas metformina apresentou interação significativamente importante. Na presença de alimentos, este medicamento tem sua absorção aumentada, além disso, influenciam diretamente na diminuição de desconforto gástrico, náusea, diarreia, dor abdominal 
Quadro 1. Interações medicamento-alimento envolvendo anti-hipertensivos e antidiabéticos prescritos no município de Caicó-RN, 2019.

\begin{tabular}{|c|c|c|c|c|c|c|}
\hline Fărmaco & $\begin{array}{l}\text { Alimento/ } \\
\text { Nutriente }\end{array}$ & Efeito & Gravidade & Situação & Tipo & Intervenção \\
\hline \multicolumn{7}{|c|}{ ANTI-HIPERTENSIVOS } \\
\hline Captopril & $\begin{array}{l}\text { Alimentos em geral, } \\
\text { principalmentericos } \\
\text { em ferro }(15,16,18)\end{array}$ & $\begin{array}{l}\text { Reduz absorção do } \\
\text { fármaco em } 30-40 \% \\
\text { (18) }\end{array}$ & Leve (15) & $\begin{array}{l}\text { Negativa } \\
(15,18)\end{array}$ & $\begin{array}{l}\text { Farmacocinética } \\
\text { (18) }\end{array}$ & $\begin{array}{l}\text { Administrar o fármaco } 1 \mathrm{~h} \text { antes } \\
\text { das refeições }(15,18)\end{array}$ \\
\hline Enalapril & $\begin{array}{l}\text { Alimentos ricos em } \\
\text { potássio }(15,18)\end{array}$ & $\begin{array}{l}\text { Risco de } \\
\text { hipercalemia, } \\
\text { podendo causar } \\
\text { arritmias }(15,18)\end{array}$ & $\begin{array}{l}\text { Moderada } \\
(15)\end{array}$ & $\begin{array}{l}\text { Negativa } \\
(18)\end{array}$ & $\begin{array}{l}\text { Farmacocinética } \\
\text { (18) }\end{array}$ & $\begin{array}{l}\text { Precaução em consumo e } \\
\text { suplementação de potássio (15) }\end{array}$ \\
\hline Atenolol & $\begin{array}{l}\text { Alimentos em geral } \\
(16,18)\end{array}$ & $\begin{array}{l}\text { Retardo da absorção } \\
\text { do fármaco }(16,18)\end{array}$ & $\begin{array}{l}\text { Moderada } \\
(16)\end{array}$ & $\begin{array}{l}\text { Negativa } \\
(18)\end{array}$ & $\begin{array}{l}\text { Farmacocinética } \\
\text { (18) }\end{array}$ & $\begin{array}{l}\text { Manter intervalo de tempo de } 1 \mathrm{~h} \\
\text { entre administração do fármaco } \\
\text { e ingesta de alimentos (16) }\end{array}$ \\
\hline Propanolol & $\begin{array}{l}\text { Alimentos } \\
\text { hiperprotéicos } \\
(15,16,18)\end{array}$ & $\begin{array}{l}\text { Aumenta } \\
\text { biodisponibilidade } \\
(15,16)\end{array}$ & $\begin{array}{l}\text { Moderada } \\
(15)\end{array}$ & $\begin{array}{l}\text { Positiva } \\
\text { (18) }\end{array}$ & $\begin{array}{l}\text { Farmacocinética } \\
\text { (18) }\end{array}$ & $\begin{array}{l}\text { Administrar medicamento com } \\
\text { dietas hiperprotéicas }(15,16)\end{array}$ \\
\hline Carvedilol & $\begin{array}{l}\text { Alimentos em geral } \\
(16,18)\end{array}$ & $\begin{array}{l}\text { Diminui risco } \\
\text { de hipotensão } \\
\text { ortostática (18) }\end{array}$ & $\begin{array}{l}\text { Moderada } \\
(16)\end{array}$ & $\begin{array}{l}\text { Positiva } \\
\text { (18) }\end{array}$ & $\begin{array}{l}\text { Farmacocinética } \\
\text { (18) }\end{array}$ & $\begin{array}{l}\text { Administrar fármaco próximo } \\
\text { às refeições (18) }\end{array}$ \\
\hline Metildopa & $\begin{array}{l}\text { Alimentos ricos em } \\
\text { ferro e folato }(15,18)\end{array}$ & $\begin{array}{l}\text { Diminuição de } \\
\text { absorção do fármaco } \\
\text { (18) }\end{array}$ & $\begin{array}{l}\text { Moderada } \\
(18)\end{array}$ & $\begin{array}{l}\text { Negativa } \\
(18)\end{array}$ & $\begin{array}{l}\text { Farmacocinética } \\
\text { (18) }\end{array}$ & $\begin{array}{l}\text { Administrar fármaco com } \\
\text { intervalo de } 2 \mathrm{~h} \text { na presença de } \\
\text { alimentos ricos em ferro (18) }\end{array}$ \\
\hline $\begin{array}{l}\text { Hidrocloro- } \\
\text { tiazida }\end{array}$ & $\begin{array}{l}\text { Alimentos em geral } \\
\text { (18) }\end{array}$ & $\begin{array}{l}\text { Aumenta absorção } \\
\text { do fármaco (18) }\end{array}$ & $\begin{array}{l}\text { A gravidade } \\
\text { referida não } \\
\text { constava } \\
\text { nas fontes } \\
\text { consultadas }\end{array}$ & $\begin{array}{l}\text { Positiva } \\
\text { (18) }\end{array}$ & $\begin{array}{l}\text { Farmacocinética } \\
\text { (18) }\end{array}$ & $\begin{array}{l}\text { Administrar fármaco associado } \\
\text { com alimentos (18) }\end{array}$ \\
\hline Furosemida & $\begin{array}{l}\text { Alimentos em geral } \\
\text { (15) }\end{array}$ & $\begin{array}{l}\text { Diminui absorção } \\
\text { do fármaco (15) }\end{array}$ & $\begin{array}{l}\text { Moderada } \\
(15)\end{array}$ & $\begin{array}{l}\text { Negativa } \\
(15)\end{array}$ & $\begin{array}{l}\text { Farmacocinética } \\
\text { (15) }\end{array}$ & $\begin{array}{l}\text { Deve-se administrar o fármaco } \\
1 \mathrm{~h} \text { antes das refeições (15) }\end{array}$ \\
\hline $\begin{array}{l}\text { Espirono- } \\
\text { lactona }\end{array}$ & $\begin{array}{l}\text { Alimentos em geral } \\
\text { e ricos em potássio } \\
\text { (18) }\end{array}$ & $\begin{array}{l}\text { Risco de } \\
\text { hiperpotassemia, } \\
\text { diminuição de } \\
\text { desconforto gástrico } \\
\text { e aumento da } \\
\text { absorção (18) }\end{array}$ & $\begin{array}{l}\text { Moderada a } \\
\text { grave (15) }\end{array}$ & $\begin{array}{l}\text { Negativa/ } \\
\text { Positiva } \\
\text { (18) }\end{array}$ & $\begin{array}{l}\text { Farmacocinética } \\
\text { (18) }\end{array}$ & $\begin{array}{l}\text { Evitar alimentos ricos em } \\
\text { potássio e quando for administrar } \\
\text { o medicamento, preferir com } \\
\text { alimentos em geral, exceto } \\
\text { aqueles ricos neste íon (18) }\end{array}$ \\
\hline Losartana & $\begin{array}{l}\text { Sem interação } \\
\text { significativamente } \\
\text { importante (15) }\end{array}$ & & & & & \\
\hline Anlodipino & $\begin{array}{l}\text { Sem interação } \\
\text { significativamente } \\
\text { importante (15) }\end{array}$ & & & & & \\
\hline \multicolumn{7}{|c|}{ ANTIDIABÉTICOS } \\
\hline Metformina & $\begin{array}{l}\text { Alimentos em geral } \\
(16,18)\end{array}$ & $\begin{array}{l}\text { Aumento da } \\
\text { absorção do fármaco } \\
\text { e diminuição } \\
\text { do desconforto } \\
\text { gastrintestinal } \\
(16,18)\end{array}$ & & $\begin{array}{l}\text { Positiva } \\
\text { (16) }\end{array}$ & $\begin{array}{l}\text { Farmacocinética } \\
\text { (16) }\end{array}$ & $\begin{array}{l}\text { Administrar fármaco associado } \\
\text { a alimentos }(16,18)\end{array}$ \\
\hline $\begin{array}{l}\text { Glibencla- } \\
\text { mida }\end{array}$ & $\begin{array}{l}\text { Sem interação } \\
\text { significativamente } \\
\text { importante (15) }\end{array}$ & & & & & \\
\hline $\begin{array}{l}\text { Insulina } \\
\text { NPH }\end{array}$ & $\begin{array}{l}\text { Sem interação } \\
\text { significativamente } \\
\text { importante (15) }\end{array}$ & & & & & \\
\hline
\end{tabular}


e inapetência causados pelo medicamento (33). Entretanto, estudos mencionam que este medicamento pode suprimir absorção de vitamina B12, aumentando risco de anemia, cansaço, falta de ar, tontura e palidez, fato este não encontrado nas bases de dados analisadas durante este estudo $(11,27,34)$.

Neste estudo, dentre as 10 interações encontradas nas bases de dados analisadas, 5 (50\%) resultam em efeitos negativos, 4 (40\%) em efeitos positivos e $1(10 \%)$ em ambos os efeitos dependendo do alimento/nutriente envolvido. Desta forma, interações medicamentosas podem ser vistas sob ópticas diferentes, repercutindo no seu manejo, pois direcionam quais intervenções devem ser adotadas para cada caso específico (35).

Seguindo esse raciocínio, na presença de alimentos, a furosemida tem sua absorção comprometida, configurando efeito negativo (15). Carvedilol tem indicação de administração próximo às refeições, pois na presença de alimentos diminui risco de hipotensão postural, configurando efeito positivo $(16,19)$. Outrossim, algumas interações podem ter características positivas e negativas dependendo do alimento/nutriente como espironolactona, que, na presença de alimentos em geral, diminui risco de desconforto gastrointestinal, mas na presença de alimentos ricos em potássio pode causar hiperpotassemia (19).

As interações também podem ser classificadas em farmacocinética, quando causada em nível de absorção, distribuição, metabolismo ou excreção, e farmacodinâmica, quando interfere diretamente no efeito clínico do medicamento (36). Neste estudo, constatou-se que todas as interações medicamento-alimento se classificam como farmacocinéticas e interferem diretamente na etapa de absorção dos tecidos biológicos para a corrente sanguínea. Desta forma, é possível identificar a intervenção ideal, nesse caso, orientar sobre o horário da tomada dos medicamentos (37).

É essencial destacar que interações medicamento-alimento podem envolver outros medicamentos que não anti-hipertensivos e antidiabéticos, daí a importância de atentar a todos os medicamentos presente nas prescrições. Neste estudo, também foram identificados nas prescrições amiodarona, ácido fólico, nisina, ácido acetilsalicílico, alendronato de sódio, digoxina, sinvastatina, omeprazol e clopidogrel.

Quanto às interações medicamento-planta medicinal, dos 14 fármacos analisados, 7 (50\%) estavam envolvidos em interações, sendo 5 anti-hipertensivos e 2 antidiabéticos (Quadro 2).

Quadro 2. Interações medicamento-planta medicinal envolvendo anti-hipertensivos e antidiabéticos prescritos no município de Caicó, RN, 2019.

\begin{tabular}{|c|c|c|c|c|c|}
\hline Fármaco & $\begin{array}{l}\text { Planta Medicinal/ } \\
\text { Parte da Planta (38) }\end{array}$ & Efeito (38) & $\begin{array}{l}\text { Situação } \\
(38)\end{array}$ & Tipo (38) & Intervenção (38) \\
\hline \multicolumn{6}{|c|}{ ANTI-HIPERTENSIVOS } \\
\hline Captopril & $\begin{array}{l}\text { Alho (Allium sativum L.) } \\
\text { / folha modificada; } \\
\text { Laranja (Citrus sp.) } \\
\text { / folha, fruto }\end{array}$ & $\begin{array}{l}\text { Potencialização do } \\
\text { efeito hipotensor }\end{array}$ & $\begin{array}{l}\text { Positiva/ } \\
\text { Negativa }\end{array}$ & Farmacodinâmica & $\begin{array}{l}\text { É importante que a } \\
\text { planta medicinal } \\
\text { seja apenas utilizada } \\
\text { após indicação de um } \\
\text { profissional de saúde. }\end{array}$ \\
\hline Propanolol & $\begin{array}{l}\text { Alho (Allium sativum L.) } \\
\text { / folha modificada; } \\
\text { Laranja (Citrus sp.) } \\
\text { / folha, fruto }\end{array}$ & $\begin{array}{l}\text { Potencialização do } \\
\text { efeito hipotensor }\end{array}$ & $\begin{array}{l}\text { Positiva/ } \\
\text { Negativa }\end{array}$ & Farmacodinâmica & $\begin{array}{l}\text { É importante que a } \\
\text { planta medicinal } \\
\text { seja apenas utilizada } \\
\text { após indicação de um } \\
\text { profissional de saúde. }\end{array}$ \\
\hline Carvedilol & Laranja (Citrus sp.) /folha, fruto & $\begin{array}{l}\text { Potencialização do } \\
\text { efeito hipotensor }\end{array}$ & $\begin{array}{l}\text { Positiva/ } \\
\text { Negativa }\end{array}$ & Farmacodinâmica & $\begin{array}{l}\text { É importante que a } \\
\text { planta medicinal } \\
\text { seja apenas utilizada } \\
\text { após indicação de um } \\
\text { profissional de saúde }\end{array}$ \\
\hline
\end{tabular}




\begin{tabular}{|c|c|c|c|c|c|}
\hline Fármaco & $\begin{array}{l}\text { Planta Medicinal/ } \\
\text { Parte da Planta (38) }\end{array}$ & Efeito (38) & $\begin{array}{l}\text { Situação } \\
\text { (38) }\end{array}$ & Tipo (38) & Intervenção (38) \\
\hline Furosemida & $\begin{array}{l}\text { 1. Chuchu [Sechium edule } \\
\text { (Jacq.) Swartz] } \\
\text { / flor, semente, raiz } \\
\text { 2. Alcachofra (Cynara scolymus L.) } \\
\text { / folha }(39,40) \\
\text { 3. Ginseng (Panax ginseng L.) } \\
\text { / raiz (41) }\end{array}$ & $\begin{array}{l}\text { 1,2. Potencialização } \\
\text { do efeito } \\
\text { hipotensor } \\
\text { 3. Aumento de } \\
\text { pressão arterial }\end{array}$ & $\begin{array}{l}\text { Positiva/ } \\
\text { Negativa }\end{array}$ & Farmacodinâmica & $\begin{array}{l}\text { É importante que a } \\
\text { planta medicinal } \\
\text { seja apenas utilizada } \\
\text { após indicação de um } \\
\text { profissional de saúde }\end{array}$ \\
\hline Anlodipino & $\begin{array}{l}\text { Alho (Allium sativum L.) } \\
\text { / folha modificada; } \\
\text { Colônia [Alpinia zerumbet (Pers.) } \\
\text { B. L. Burtt \& R. M. Sm] (38) } \\
\text { / folha; } \\
\text { Capim santo [Cymbopogon citratus } \\
\text { (D.C.) Stapf] (38) } \\
\text { / folha seca ou fresca, raiz } \\
\text { rizomatosa }\end{array}$ & $\begin{array}{l}\text { Potencialização do } \\
\text { efeito hipotensor, } \\
\text { com alto risco de } \\
\text { hipotensão para } A \text {. } \\
\text { zerumbet }\end{array}$ & $\begin{array}{l}\text { Positiva/ } \\
\text { Negativa }\end{array}$ & Farmacodinâmica & $\begin{array}{l}\text { É importante que a } \\
\text { planta medicinal } \\
\text { seja apenas utilizada } \\
\text { após indicação de um } \\
\text { profissional de saúde }\end{array}$ \\
\hline $\begin{array}{l}\text { Hidrocloro- } \\
\text { tiazida }\end{array}$ & $\begin{array}{l}\text { Alcachofra (Cynara scolymus L.) } \\
\text { / folha }(39,40)\end{array}$ & $\begin{array}{l}\text { Potencialização do } \\
\text { efeito hipotensor }\end{array}$ & $\begin{array}{l}\text { Positiva/ } \\
\text { Negativa }\end{array}$ & Farmacodinâmica & $\begin{array}{l}\text { É importante que a } \\
\text { planta medicinal } \\
\text { seja apenas utilizada } \\
\text { após indicação de um } \\
\text { profissional de saúde }\end{array}$ \\
\hline Atenolol & $\begin{array}{l}\text { Sem interação significativamente } \\
\text { importante }\end{array}$ & & & & \\
\hline Enalapril & $\begin{array}{l}\text { Sem interação significativamente } \\
\text { importante }\end{array}$ & & & & \\
\hline $\begin{array}{l}\text { Espirono- } \\
\text { lactona }\end{array}$ & $\begin{array}{l}\text { Sem interação significativamente } \\
\text { importante }\end{array}$ & & & & \\
\hline Losartana & $\begin{array}{l}\text { Sem interação significativamente } \\
\text { importante }\end{array}$ & & & & \\
\hline Metildopa & $\begin{array}{l}\text { Sem interação significativamente } \\
\text { importante }\end{array}$ & & & & \\
\hline
\end{tabular}

\section{ANTIDIABÉTICOS}

\begin{tabular}{|c|c|c|c|c|c|}
\hline \multicolumn{6}{|c|}{ ANTIDIABÉTICOS } \\
\hline $\begin{array}{l}\text { Glibencla- } \\
\text { mida }\end{array}$ & $\begin{array}{l}\text { Alho (Allium sativum L.) } \\
\text { / folha modificada; } \\
\text { Pata de Vaca (Bauhinia forficata L.) } \\
\text { / folha, casca }\end{array}$ & $\begin{array}{l}\text { Estimula a secreção } \\
\text { de insulina }\end{array}$ & $\begin{array}{l}\text { Positiva/ } \\
\text { Negativa }\end{array}$ & Farmacodinâmica & $\begin{array}{l}\text { É importante que a } \\
\text { planta medicinal } \\
\text { seja apenas utilizada } \\
\text { após indicação de um } \\
\text { profissional de saúde }\end{array}$ \\
\hline \multirow{4}{*}{$\begin{array}{l}\text { Insulina } \\
\text { NPH }\end{array}$} & $\begin{array}{l}\text { 1. Alho (Allium sativum L.) } \\
\text { / folha modificada; }\end{array}$ & $\begin{array}{l}\text { 1. Diminuição } \\
\text { excessiva dos } \\
\text { níveis de glicose } \\
\text { sanguínea; }\end{array}$ & \multirow{4}{*}{$\begin{array}{l}\text { Positiva/ } \\
\text { Negativa }\end{array}$} & \multirow{4}{*}{ Farmacodinâmica } & \multirow{4}{*}{$\begin{array}{l}\text { É importante que a } \\
\text { planta medicinal } \\
\text { seja apenas utilizada } \\
\text { após indicação de um } \\
\text { profissional de saúde }\end{array}$} \\
\hline & $\begin{array}{l}\text { 2. Pata de Vaca } \\
\text { (Bauhinia forficata L.) } \\
\text { / folha, casca; }\end{array}$ & $\begin{array}{l}\text { 2. Estímulo à } \\
\text { secreção de } \\
\text { insulina; }\end{array}$ & & & \\
\hline & $\begin{array}{l}\text { 3. Picão (Bidens pilosa L.) } \\
\text { / toda planta; }\end{array}$ & $\begin{array}{l}\text { 3. Estímulo à } \\
\text { produção de } \\
\text { insulina; }\end{array}$ & & & \\
\hline & $\begin{array}{l}\text { 4. Melão de São Caetano } \\
\text { (Momordica charantia L.) (38) / } \\
\text { folha, fruto }\end{array}$ & $\begin{array}{l}\text { 4. Potencialização } \\
\text { do efeito } \\
\text { hipoglicemiante. }\end{array}$ & & & \\
\hline Metformina & $\begin{array}{l}\text { Sem interação significativamente } \\
\text { importante }\end{array}$ & & & & \\
\hline
\end{tabular}


Neste estudo, todas as interações foram de caráter positivo/negativo, dependendo da dose e frequência de administração do medicamento e da planta medicinal. Ademais, diversas plantas apresentam efeitos tóxicos, ou seja, a ideia de que tudo que é natural é inócuo precisa ser revista, a fim de prevenir eventos adversos (42). Também foi constatado que todas as interações foram de caráter farmacodinâmico, ou seja, podem interferir no efeito clínico tanto do fármaco quanto da planta medicinal (35). Além disso, estas interações são consideradas sinérgicas, pois o efeito da interação é maior que o do medicamento isolado (43). Não foi possível classificar estas interações de acordo com sua gravidade, em decorrência da carência de estudos referentes ao assunto.

Propranolol e captopril podem interagir com alho, que pode potencializar o efeito hipotensor destes fármacos, dependendo da quantidade de alho ingerida (44). Alcachofra apresenta ação diurética e, quando administrada com fármacos diuréticos como hidroclorotiazida e furosemida, pode gerar drástica diminuição do volume sanguíneo, consequentemente, queda da pressão arterial $(39,40)$.

$\mathrm{Na}$ literatura, alho e pata da vaca são bem descritas como indicadas para diabetes, entretanto, é grande a probabilidade de interações com medicamentos. Quando estas plantas são usadas concomitantemente com antidiabéticos, podem potencializar seu efeito hipoglicemiante, podendo resultar em hipoglicemia leve a moderada, interferindo diretamente na segurança do tratamento $(39,45)$.

Diante desse contexto, fica claro que portadores de diabetes e hipertensão não devem utilizar estas plantas medicinais sem orientações de um profissional capacitado, pois podem contribuir para hipoglicemia e hipotensão, trazendo sérios riscos à segurança do paciente. Portanto, cabe aos profissionais de saúde contribuir para assegurar efetividade e segurança da farmacoterapia (46).

Seguindo esse raciocínio, reflete-se sobre o papel do farmacêutico em interações medicamento-alimento/medicamento-planta medicinal, cabendo a este profissional de saúde e especialista do medicamento o dever de identificar, prevenir e resolver problemas relacionados a medicamentos, contribuindo para garantir a efetividade terapêutica e a segurança do paciente (47).

\section{CONCLUSÃO}

Considerando os $10(71,4 \%)$ medicamentos envolvidos, das interações medicamento-alimento/nutriente, 6 são consideradas negativas (1 leve, 4 moderadas e 1 moderada a grave) e 4 positivas. Considerando os 7 (50\%) medicamentos envolvidos, das interações medicamento-planta medicinal, $100 \%$ são consideradas positivas/ negativas a depender de dose e frequência de administração do medicamento e da planta medicinal.

Diante do exposto, depreende-se a importância de desenvolvimento de mais estudos sobre este tema. Além disso, espera-se sensibilização e instrumentalização de profissionais de saúde e usuários acerca do manejo adequado da terapia farmacológica, a fim de usufruir das interações benéficas bem como evitar os impactos negativos sobre a efetividade terapêutica e a segurança do paciente que algumas interações podem causar.

\section{REFERÊNCIAS}

1. WHO. From burden to "best buys": reducing the economic impact of NCDs in low-and middle-income countries. Geneva: World Health Organization. 2011.

2. Malta DC, França E, Abreu DMX, Perillo RD, Salmen MC, Teixeira RA, Passos V, Sousa MFM, Mooney M, Naghavi M. Mortalidade por doenças não transmissíveis no Brasil, de 1990 a 2015, segundo estimativas do estudo Global Burden of Disease. São Paulo Med. J.
2017;135(3):213-221. DOI: 10.1590/1516-3180.2016. 0330050117.

3. SBD. Diretrizes da Sociedade Brasileira de Diabetes (2019-2020). 2019.

4. SBC, SBH, SBN.. Diretrizes Brasileiras de Hipertensão Arterial - 2020. Sociedade Brasileira de Cardiologia/ Sociedade Brasileira de Hipertensão/Sociedade Brasileira de Nefrologia. Arq Bras Cardiol. 2020. 
5. Siebra KLDAB, Arraes JCC, Santos DB, Nascimento $\mathrm{CH}$, Leandro IVA, Basílio CAS, Honorato JM, Noronha JWC, Castro APR, Medeiros KMF. Promovendo saúde: um elo de cuidados no tratamento não medicamentoso de doenças crônicas na terceira idade. Rev. Interfaces: Saúde, Humanas e Tecnologia. 2019;7(1):250-254. DOI: 10.16891/2317-434X.v7.e1.a2019.pp250-254.

6. Moura MRL, Reyes FGR. Interação fármaco-nutriente: uma revisão. Rev. Nutr. 2002;15(2):223-238. DOI: 10.1590/S1415-52732002000200011.

7. Gomez R, Venturini CD. Interação entre alimentos e medicamentos. Porto Alegre: Letra e Vida. 2009.

8. Jáuregui-Garrido B, Jáuregui-Lobera I. Interactions between antihypertensive drugs and food. Nutr Hosp. 2012;27(6):1866-1875. DOI: 10.3305/nh.2012.27.6.6127.

9. Cock IE. The safe usage of herbal medicines: counterindications, cross-reactivity and toxicity. Phoog Commn. 2015;5(1):1-38. DOI:10.5530/pc.2015.1.2.

10. Mamindla S, Prasad KVSRG, Koganti B. Herb-drug interactions: an overview of mechanisms and clinical aspects. Int J Pharm Sci Res. 2016;7(9):3576-3586. DOI: 10.13040/IJPSR.0975-8232.7(9).3576-86.

11. Sandri M, Gewehr DM, Huth A, Moreira AC. Uso de medicamentos e suas potenciais interações com alimentos em idosos institucionalizados. Sci. Med, 2016;26(4):1-8. DOI: 10.15448/1980-6108.2016.4.23780.

12. Lopes EM, Carvalho RBND, Freitas RMD. Análise das possíveis interações entre medicamentos e alimento/ nutrientes em pacientes hospitalizados. Einstein. 2010; 8(3):298-302. DOI: 10.1590/s1679-45082010ao1672.

13. Antunes AO, Lo Prete AC. O papel da atenção farmacêutica frente às interações fármaco-nutriente. Infarma Cienc Farm. 2014;26(4):208-214. DOI: 10.14450/23189312.v26.e4.a2014.pp208-214.

14. IBGE. IBGE CidadesInstituto Brasileiro de Geografia e Estatística. Disponível em: https://cidades.ibge.gov.br/ brasil/rn/caico/panorama [acessado em 18 de dezembro de 2020].

15. Micromedex Healthcare. Disponível em: http://www. micromedex.com/products/hcs/ [acessado em novembro de 2020].

16. Drug-Reax System. Disponível em: http://www. thomsonhc.com [acessado em novembro de 2020].

17. UpToDate. Disponível em: https://www.uptodate.com/ contents/drug-prescribing-for-older-adults [acessado em novembro de 2020].

18. ANVISA. Agência Nacional de Vigilância Sanitária. Bulário eletrônico. [acessado em 23 de julho de 2020]. Disponível em: http://www4.anvisa.gov.br/BularioEletronico.

19. Ribeiro DR, Calixto DM, Silva LL, Alves RPCN, Souza LMC. Prevalência de diabetes mellitus e hipertensão em idosos. Rev Artigos. Com. 2020;14:1-6.
20. Rêgo ADS, Soares AC, Silva PA, Fernandes CAM, Baldissera VDA, Radovanovic CAT. Perfil medicamentoso de pessoas com hipertensão arterial. Cienc Cuid Saude. 2019;18(4):1-9. DOI: 10.4025/cienccuidsaude. v18i4.46518.

21. Coneglian K, Guedes BLM, Biazon ACB. Perfil farmacoepidemiológico dos portadores de diabetes mellitus cadastrados em uma unidade básica de saúde de Barbosa Ferraz, Paraná, Brasil. Rev Iniciare. 2017; 2(1):108-118.

22. Augusto DK, David L, Oliveira DOPS, Trindade TG, Lermen Junior N, Poli Neto P. Quantos médicos de família e comunidade temos no Brasil? Rev Bras Med Fam Comunidade. 2018;13(40):1-4. DOI: 10.5712/ rbmfc13(40)1695.

23. Santos MAS. Medicina de Família e Comunidade: um médico para todas as pessoas. Rev Med. 2012;91:39-42. DOI: 10.11606/issn.1679-9836.v91i0p39-42.

24. Afonso MP, Shimizu HE, Merchan-Hamann E, Ramalho WM, Afonso T. Association between hospitalisation for ambulatory care-sensitive conditions and primary health care physician specialisation: a cross-sectional ecological study in Curitiba (Brazil). BMJ Open. 2017;7(12):1-8. DOI: 10.1136/bmjopen-2016-015322.

25. Jaco BR, Norman AH. A filosofia da Medicina de Família e Comunidade segundo Ian McWhinney e Roger Neighbour. Rev Bras Med Fam Comunidade. 2020;15(42):1-9. DOI: 10.5712/rbmfc15(42)1991.

26. CAICÓ. Secretaria Municipal de Saúde. Relação Municipal de Medicamentos Essenciais. 2014.

27. Pereira MTL, Queiroz MP, Bertozzo CCMS, Barbosa MQEA, Campos RS, Pinto MLM. Interações fármaconutriente de anti-hipertensivos e antidiabéticos prescritos no Hospital Universitário Alcides Carneiro. Res, Soc Develop 2018;9(7):1-32. DOI: 10.33448/rsd-v9i7.3802.

28. Campos MG, Castilho MC, Santos LM, Ramos FJ. Medicamentos, alimentos e plantas: as interacções esquecidas? Lisboa: Hollyfar, Marcas e Comunicação. 2014.

29. Peixoto JS, Salci MA, Radovanovic CAT, Salci TP, Torres MM, Carreira L. Riscos da interação droga-nutriente em idosos de instituição de longa permanência. Rev. Gaúch. Enferm. 2012;33(3):156-164. DOI: 10.1590/S198314472012000300021.

30. Linhares AO, Cesar JA. Suplementação com ácido fólico entre gestantes no extremo Sul do Brasil: prevalência e fatores associados. Cien Saude Colet. 2017;22(2):535542. DOI: 10.1590/1413-81232017222.06302016.

31. Raebel MA, Ellis JL, Carroll NM, Bayliss, EA, McGinnis B, Schroeder EB, Steiner JF. Characteristics of patients with primary non-adherence to medications for hypertension, diabetes, and lipid disorders. J Gen Intern Med. 2012;27(1):57-64. DOI: 10.1007 / s11606011-1829-z. 
32. Franklin B, Trindade DF, Assunção Júnior DA, Rodrigues MQ, Farias RAF. Interações da furosemida com nutrientes. In: 12. Congresso Brasileiro de Medicina de Família e Comunidade. 2013. Belém: Sociedade Brasileira de Medicina de Família e Comunidade; 2013.

33. EBSERH. Empresa Brasileira de serviços Hospitalares. Medicamentos padronizados para administração oral: guia para administração racional. [acessado em 18 de dezembro de 2020]. Disponível em: http://www2.ebserh. gov.br/documents/220250/1293244/GUIA+F\%C3\%81 RMACO+X+NUTRIENTE+EM+CONSTRU $\% \mathrm{C} 3 \%$ $87 \%$ C3\%83O.pdf/ebe6b0bd-321d-4983-bb61-89c33d$22 \mathrm{f} 46 \mathrm{e}$.

34. Chapman LE, Darling AL, Brown JE. Association between metformin and vitamin B12 deficiency in patients with type 2 diabetes: a systematic review and meta-analysis. Diabetes Metab. 2016;42(5):316-327. DOI: $10.1016 /$ j.diabet.2016.03.008.

35. Lopes EM, Oliveira EAR, Lima LHO, Formiga LMF, Freitas RM. Interações fármaco-alimento/nutriente potenciais em pacientes pediátricos hospitalizados. Rev Ciênc Farm Básica Apl. 2013;34(1):131-135.

36. Samano MTSM, Méndez JLS. Interacciones alimenteo/ medicamento. Inf Ter Sist Nac Salud. 2011;35(1):3-12.

37. Tozer TN, Rowland M. Introdução à farmacocinética e à farmacodinâmica: as bases quantitativas da terapia farmacológica. Porto Alegre: Artmed. 2009.

38. Ataliba FJB. Interações planta x medicamento alopático no tratamento de diabetes e hipertensão arterial. [Monografia]. Cuité: Universidade Federal de Campina Grande. 2016.

39. Dias ECM, Trevisan DD, Nagai SC, Ramos NA, Silva EM. Uso de fitoterápicos e potenciais riscos de interações medicamentosas: reflexões para prática segura. Rev. Baiana Saúde Pública. 2018;41(2):297-307. DOI: 10.22278/2318-2660.2017.v41.n2.a2306.
40. Noldin VF, Cechinel Filho V, Monache FD, Benassi JC, Christmann IL, Pedrosa RC, Yunes RA. Composição química e atividades biológicas das folhas de Cynara scolymus L. (alcachofra) cultivada no Brasil. Quím Nova. 2003;26(3):331-334. DOI: 10.1590/S0100-4042 2003000300008 .

41. Tachjian A, Maria V, Jahangir A. Use of herbal products and potential interactions in patients with cardiovascular diseases. J Am Coll Cardiol. 2010;55(6):515-525. DOI: 10.1016/j.jacc.2009.07.074.

42. Santos RL, Guimaraes GP, Nobre MSC, Portela AS. Análise sobre a fitoterapia como prática integrativa no Sistema Único de Saúde. Rev. Bras. Plantas Med. 2011;13(4):486-491. DOI: 10.1590/S1516-05722011000 400014.

43. Casanova LM, Costa SS. Interações sinérgicas em produtos naturais: potencial terapêutico e desafios. Rev. Virtual Quim. 2017;9(2):575-595. DOI: 10.21577/19846835.20170034 .

44. Brito JF. Possíveis interações entre fitoterapias e fármacos observados em grupos de Hiperdia, na região do vale do Jamari-RO. [Monografia]. Ariquemes: Faculdade de Educação e Meio Ambiente. 2018.

45. Godoi JP, Guterres LS, Bruscato CM, Quevedo EG, Farias FM, Uso de plantas medicinais por indivíduos diabéticos em Uruguaiana: possíveis interações medicamentosas. In: 7. Salão Internacional de Ensino, Pesquisa e Extensão. 2015. Alegrete: Universidade Federal do Pampa; 2015.

46. Trindade MT, Bezerra NN, Starling PS, Viana EDSM, Torres SAM, Gusman GS. Atenção farmacêutica na fitoterapia. Rev Cient Univiçosa. 2018;10(1):1074-1080.

47. Couto ARD. Interações alimento-medicamento no tratamento da hipertensão. [Tese]. Coimbra: Universidade de Coimbra. 2018. 